\title{
Current Issues in Bariatric Surgery for Adolescents with Severe Obesity: Durability, Complications, and Timing of Intervention
}

\author{
Soo Min Ahn* \\ Department of Surgery, Bariatric and Metabolic Surgery Center, Pediatric Specialized Center, Hallym University Sacred Heart Hospital, Anyang, Korea
}

Conventional nonsurgical management of severe obesity in the pediatric population and adolescents has focused on a multidisciplinary approach involving diet, exercise, behavioral modification, and to some extent, pharmaceuticals. Although nonsurgical strategies provide a certain degree of effective weight reduction, most of the severely obese adolescents suffer from a high relapse rate. In recent studies, long-term outcomes of bariatric surgery for severely obese adolescents have shown sustainable effects on weight loss and resolution of related comorbidities, such as hypertension and type 2 diabetes mellitus in this vulnerable age group. Notably, the role and practical benefits of bariatric surgery as a multidisciplinary therapeutic approach to adolescent obesity is gaining attention and acceptance. However, a surgical approach has many obstacles that prevent the timely evaluation and optimal intervention for adolescent obesity and its comorbidities. In the present review, the latest data on long-term outcomes after bariatric surgery for severely obese adolescents in terms of durability, effects on metabolic risk factors, complications, and optimal timing were summarized. The results showed the sustainability of weight loss and comorbidity resolution in adolescents following bariatric surgery. In addition, earlier surgery in patients without an extremely high body mass index increases the likelihood of a healthier life in adulthood. This review can help clarify the beneficial effects of bariatric surgery on weight reduction and resolution of comorbidities in severely obese adolescents and remove the barriers to referral of adolescents for bariatric surgery.

Received December 6, 2019

Reviewed January 16, 2020

Accepted February 20, 2020

*Corresponding author

Soo Min Ahn

https://orcid.org/0000-0002-2854-5032

Department of Surgery, Bariatric and Metabolic Surgery Center, Pediatric Specialized Center, Hallym University Sacred Heart Hospital, 22 Gwanpyeong-ro 170beon-gil, Dongan-gu, Anyang 14068, Korea Tel: $+82-31-380-1825$

Fax: +82-31-380-1619

E-mail:smahn@hallym.ac.kr

Key words: Obesity, Bariatric surgery, Adolescents, Durability, Complications

\section{INTRODUCTION}

The prevalence of obesity in the pediatric population (body mass index $[\mathrm{BMI}]>95$ th percentile for age and sex) is $18 \%$ in the United States. ${ }^{1,2}$ Extreme obesity, defined as a BMI > 99.5 percentile, has increased up to $6 \%$. ${ }^{1,2}$ The socioeconomic burden for the management of obesity and related disorders in the pediatric population should be discussed as part of a major health care policy and extensively investigated because ineffectively controlled childhood obesity carries substantial healthcare implications. ${ }^{3,4}$ These findings justify that an active obesity management strategy should be imple- mented in the pediatric population and adolescents.

In general, obese children remain obese in adulthood. In five previous longitudinal U.S. studies, $57 \%$ of children were reportedly at risk of being obese by the age of 35 years. Furthermore, obesity will begin before adulthood in $50 \%$ of these children. ${ }^{5}$ Studies on the long-term effects of obesity in teenagers clearly demonstrate that obese children and adolescents are at risk of premature mortality and significant adverse health consequences, such as type 2 diabetes mellitus (T2DM) and hypertension., ${ }^{3,6}$ The morbidity related to adolescent obesity is substantial and even more aggressive. ${ }^{3,5,6}$

Currently, no singularly effective medical option is available for

Copyright @ 2020 Korean Society for the Study of Obesity

(a) This is an Open Access article distributed under the terms of the Creative Commons Attribution Non-Commercial License (https://creativecommons.org/licenses/by-nc/4.o/) which permits unrestricted non-commercial use, distribution, and reproduction in any medium, provided the original work is properly cited. 
sustainable weight reduction in severely obese adolescents. Approximately $3 \%-44 \%$ of patients on weight-loss medication experience a certain degree of adverse reactions, ${ }^{7-9}$ sometimes leading to additional hospital admissions and the aggravation of psychiatric problems. These findings emphasize the need for appropriate and timely surgical intervention because severely obese teenagers are likely to remain severely obese with premature mortality and intractable morbidity. ${ }^{5}$

Recently, the role of bariatric surgery in severely obese adolescents has received more attention, however, the surgery has not yet been universally accepted. Referring practitioners still disagree on the safety and long-term efficacy of surgical intervention in teenagers which creates a reluctance to refer. However, based on recent evidence regarding safety and efficacy of bariatric surgery in adolescents, revisiting the role of surgical intervention is warranted due to the high obesity epidemic in teenagers and the strong controversial opinions.

In general, recognition of the potential benefits of adolescent bariatric surgery is evolving; however, only a decade ago almost half of referring practitioners responded they would not refer their adolescent patients with severe obesity to bariatric surgeons regardless of specialty and years since board certification. According to a survey in 2007, the respondents considered 18 years as the minimum acceptable age for referral. In addition, the BMI percentiles reported appropriate for bariatric surgery referral ranged widely. Surprisingly, a third of the respondents had no knowledge of the recommendations regarding bariatric surgery as a treatment option in a multidisciplinary strategy for adolescent obesity from the expert literature such as the American Academy of Pediatrics. ${ }^{10}$ More recently, the issues on the perception of bariatric surgery and practice of bariatric surgery in adolescents were reexamined ${ }^{10}$ and only $15 \%$ of respondents were still claiming they would not refer adolescents of any age for bariatric surgery. Referring pediatricians and family physicians who have fewer years of practice are less likely to avoid recommending bariatric surgery and more likely to consider bariatric surgery as part of a multidisciplinary obesity treatment strategy. Consequently, greater exposure to the concept of bariatric surgery for adolescents may have led to a potential shift in opinion.

There are significant and rational concerns, especially regarding safety, in this vulnerable age group. Given its irreversible and inva- sive nature, whenever pediatric bariatric surgery is considered, several issues in terms of effectiveness, sustainability, procedure-specific complications, teenage specific adverse effects, and optimal timing of intervention as well as ethics arise. These issues require thoughtful consideration and justification based on evidence from well-designed studies.

In the present review, recent data on long-term ( $>5$ years) outcomes after bariatric surgery for severely obese adolescents were summarized, specifically regarding durability, complications, and optimal timing for surgery. The conclusions drawn from this review can help overcome the obstacles to adopting bariatric surgery as an effective therapeutic modality for severely obese adolescents.

\section{DURABILITY}

Referring severely obese adolescents to bariatric surgeons may provide substantial benefits. ${ }^{11}$ A multicenter analysis of 1-year outcomes after Roux-en-Y gastric bypass (RYGB) for adolescents with severe obesity showed a mean BMI reduction of $37 \%$ and a substantial reduction in the rate of metabolic syndrome, including a resolution of T2DM in approximately $50 \%-100 \%$ of patients, resolution of dyslipidemia in 23\%-100\%, and hypertension in 54\%$100 \% .{ }^{12}$ In the Teen-Longitudinal Assessment of Bariatric Surgery (Teen-LABS) study, a multicenter prospective trial which enrolled teenagers at a mean age of 17 years, showed the mean BMI reduced to $39 \mathrm{~kg} / \mathrm{m}^{2} 3$ years after surgery from $52.5 \mathrm{~kg} / \mathrm{m}^{2}$ preoperatively. Despite the substantial reduction in BMI, the reduced BMI remains indicative of a severely obese population. ${ }^{13}$

A primary weakness of a lifestyle modification strategy is the lack of a sustained effect on weight loss, ${ }^{14,15}$ which is a similar concern regarding bariatric surgery for severely obese adolescents. Consequently, determining the role of adolescent bariatric surgery and referral of adolescents to bariatric surgeons has been hindered due to lack of evidence on durability from long-term studies. Meaningful evidence is beginning to emerge from well planned and methodic clinical efforts and research trials. The outcomes of long-term $(>5$ years) prospective multicenter trials have consistently shown sustainable weight reduction and compelling resolution rates of comorbidities. ${ }^{16-18}$ In the Swedish Adolescent Morbid Obesity Surgery (AMOS) study, a significantly lower BMI was observed post- 
Table 1. Long-term outcomes of multicenter prospective trials of bariatric surgery in adolescents

\begin{tabular}{|c|c|c|c|c|c|c|c|c|}
\hline \multirow{2}{*}{ Author (year) } & \multirow{2}{*}{ Study } & \multirow{2}{*}{$\begin{array}{c}\text { Followed-up/ } \\
\text { enrolled patient (n) }\end{array}$} & \multirow{2}{*}{$\begin{array}{l}\text { Age } \\
\text { (yr) }\end{array}$} & \multirow{2}{*}{$\begin{array}{c}\text { BMl at the } \\
\text { baseline }\left(\mathrm{kg} / \mathrm{m}^{2}\right)\end{array}$} & \multirow{2}{*}{$\begin{array}{c}\text { Change in BMl } \\
(\%)\end{array}$} & \multicolumn{3}{|c|}{ Remission rate of comorbidity (\%) } \\
\hline & & & & & & T2DM & Hypertension & Dyslipidemia \\
\hline Olbers et al. $(2017)^{16}$ & AMOS & $81 / 81$ & $16.5(13-18)$ & $45.5 \pm 6.1$ & -29 & 100 & 92 & 83 \\
\hline Inge et al. $(2017)^{17}$ & FABS 5+ & $58 / 58$ & $17.1(13-21)$ & $58.5 \pm 10.1$ & -29 & 88 & 76 & 64 \\
\hline Inge et al. (2019)18 & Teen-LABS & $149 / 161$ & $17.0(<18)$ & $54 \pm 10$ & -26 & 86 & 68 & - \\
\hline
\end{tabular}

Values are presented as median (range) or mean \pm standard deviation.

BMI, body mass index; T2DM, type 2 diabetes mellitus; AMOS, Swedish Adolescent Morbid Obesity Surgery; FABS 5+, Follow-up of Adolescent Bariatric Surgery at 5 Plus Years; Teen-LABS, Teen-Longitudinal Assessment of Bariatric Surgery.

operatively compared with age-matched medically-managed controls. Furthermore, cardiovascular risk factors substantially improved after RYGB (Table 1), including glucose tolerance, blood pressure, and plasma lipid concentration. ${ }^{16}$ In particular, all patients experienced T2DM remission, which was better than any results previously reported for adults. ${ }^{19}$

The outcomes of adolescent bariatric surgery 5-12 years after RYGB were investigated in the Follow-up of Adolescent Bariatric Surgery at 5 Plus Years (FABS 5+) study, a recent long-term multicenter prospective trial. ${ }^{17}$ In that study, mean baseline BMI was reduced postoperatively by $29 \%$. The remission rate of T2DM and resolution rate of hypertension were $88 \%$ and $76 \%$, respectively (Table 1), which corroborates the findings of studies with shorter follow-up periods in the literature. ${ }^{8,13,20}$ In the latest long-term report from the Teen-LABS study, the authors reported an obvious durable weight reduction of $26 \%$ in adolescents; the T2DM and hypertension revision rates were $86 \%$ and $68 \%$, respectively (Table 1 ).

The favorable outcomes of adolescent bariatric surgery were confirmed when postoperative results of bariatric surgery were compared between adolescents and adults. In the Teen-LABS study, ${ }^{18} 5$ years after surgery, $60 \%$ of adolescents maintained a weight reduction $\geq 20 \%$, which was slightly less than in adults. Conversely, 4\% (95\% confidence interval, 2\%-9\%) of adolescents maintained a weight reduction $<5 \%$, which is slightly higher than in adults. Therefore, long-term periodic clinical surveillance for individual compliance and specialized care with a relevant solution is essential for this vulnerable age group.

\section{EFFECTS ON METABOLIC RISK FACTORS}

The parameters of cardiac and systemic circulatory function improve dramatically and are maintained long-term after bariatric sur- gery in adolescents. Echocardiography showed myocardial function significantly improved after adolescent bariatric surgery. ${ }^{21}$ An essential echocardiographic finding after RYGB in adolescents was improvement of left ventricular hypertrophy which led to improved diastolic function of the left ventricle. ${ }^{21}$ The proportion of adolescents with hypertension decreased from $43.6 \%$ at baseline to $15.5 \%$ at 3 years. Furthermore, the proportion of adolescents with dyslipidemia significantly decreased (29.4\% vs. $75.2 \%){ }^{13}$

Bariatric surgery showed significant and sustainable antidiabetic effects in adolescents, similar to adults. The proportion of adolescents with $\mathrm{T} 2 \mathrm{DM}$ was reduced from $12.6 \%$ at baseline to $0.6 \%$ at 3 years postoperatively in the Teen-LABS study. Based on this finding, timely bariatric surgery in adolescents will likely reverse the risk of full development of T2DM. When comparing the 30 participants who underwent bariatric surgery in Teen-LABS to the 63 medically-treated participants in the Treatment Options of Type 2 Diabetes in Adolescents and Youth (TODAY) study, hemoglobin Alc levels worsened from $6.4 \%$ to $7.8 \%$ in the medically-treated participants in the TODAY study; however, hemoglobin Alc improved from $6.8 \%$ to $5.5 \%$ among participants who underwent bariatric surgery in the Teen-LABS study over a 2 -year period. In the TODAY study, adolescents had been randomly assigned to medical therapy, including metformin with or without rosiglitazone or lifestyle counseling. Insulin was administered to participants in the TODAY study whose T2DM worsened over time during the study. BMI increased by $3.7 \%$ in the TODAY participants but was reduced by $29 \%$ in the Teen-LABS participants. ${ }^{18}$

The benefits of bariatric surgery may be more obvious in adolescents than in adults. In adults with severe obesity, T2DM remission has been reported in $42 \%-68 \%$ of patients 5 years after bariatric surgery. ${ }^{18,19}$ Conversely, a collective review of T2DM remission rates in long-term multicenter prospective studies of 35 diabetic 
adolescents showed approximately $89 \%$ of the patients achieved remission 5 years after bariatric surgery. ${ }^{16-18}$

\section{COMPLICATIONS}

A measurable rate of major and minor complications are associated with bariatric surgery, irrespective of the type of procedure. In general, bariatric surgeries are well tolerated with a minimal risk of serious perioperative complications. ${ }^{22-24}$ In the Teen-LABS study, complications requiring reoperation, causing harmful sequalae, or leading to life-threatening conditions occurred in $9.3 \%, 4.5 \%$, and $7.1 \%$ of patients after RYGB, sleeve gastrectomy (SG), and adjustable gastric banding (AGB), respectively. ${ }^{25}$ Minor complications not requiring additional intervention, such as additional laboratory sampling, testing, or unplanned nutritional support occurred in $16.8 \%, 11.9 \%$, and $7.1 \%$ of patients after RYGB, SG, and AGB, respectively. ${ }^{25}$ The most frequent minor complications were surgical site infections and urinary tract infections. ${ }^{8}$ The National Surgical Quality Improvement Program in the Unites States reported an overall immediate postoperative ( $<30$ days) complication rate of $2.4 \%$ in patients $18-21$ years of age. ${ }^{26}$ The major complication of anastomotic or staple line dehiscence occurred in $2 \%-5 \%$ and $0 \%-$ $20 \%$ of RYGB and SG patients, respectively. ${ }^{13,17,25}$ These complications remain a serious concern, although most patients enjoy benefits from the surgery and do not experience any adverse events. However, the benefits of bariatric surgery should be compared to the risks of not undergoing the surgery.

To the best of our knowledge, less than six deaths have been reported after bariatric surgery in adolescents to date, and none were associated with a procedure-related complication..$^{13,16-18}$ Among the 242 patients in the Teen-LABS study, no mortality occurred during the immediate postoperative period ( $<30$ days). ${ }^{13}$ In the FABS 5+ study, the authors acknowledged the significance of three mortality cases $(1.9 \%)$ in adolescents ( 2 per 500 person-years). However, the mortality rate was not significantly different compared with the adult cohort (1.8\%). ${ }^{17}$ Death in the adolescents occurred several years postoperatively and were not directly attributable to the surgery. One mortality resulted from suspected sepsis in a patient with type 1 diabetes mellitus, and two were consistent with drug overdose.
Regarding the immediate postoperative unexpected surgical intervention, recent National Surgical Quality Improvement Program data showed an immediate postoperative reoperation rate of $1.5 \%$ after bariatric surgery in adolescents and a readmission rate of $4.1 \%,{ }^{27}$ these rates are not overly high compared with other major abdominal surgeries. Furthermore, during the longer-term followup period, the reoperation rate for any reason was approximately $13 \%$ during the 3-year follow-up, as reported in the Teen-LABS study. ${ }^{13,25,28}$ The cohort showed a relatively high intraabdominal reoperation rate in adolescents compared with adults over the 5-year period ( $20 \%$ vs. $16 \%)$. The most common cause of reoperation was gallstones, followed by intestinal obstruction..$^{18} \mathrm{~A}$ high likelihood of reoperation after bariatric surgery in adolescents over a 5 -year period (25\%) was also reported in the AMOS study. ${ }^{16}$ Further examination of the surgery outcomes could partly explain the higher rate of reoperation in the adolescent cohort compared with the LABS adult cohort over the long-term follow-up. ${ }^{18}$ However, bariatric surgeries are performed much more frequently in adults. Notably, there have been recent advances in surgical techniques in adult bariatric surgery, including the effective and secure closure of mesenteric defects during RYGB and postoperative use of choleretics during the rapid weight reduction period. These factors may have resulted in patients avoiding the need for corrective surgery during the longer-term follow-up. ${ }^{18}$

The need for postoperative micronutrient supplementation is a concern. Subnormal levels of micronutrients are frequently observed in the adolescent bariatric surgery group. ${ }^{18}$ These findings are highly prevalent in obese adolescents and become more prevalent after bariatric surgery. ${ }^{16,18}$ In the AMOS study, $72 \%$ of subjects had some degree of nutritional deficiency associated with RYGB (Table 2), with anemia and vitamin D deficiencies observed in $32 \%$ and $30 \%$ of the patients, respectively, during the postoperative period of $>5$ years. ${ }^{16}$ At 2 years, subnormal micronutrient levels also appeared to occur in the AMOS, FABS 5+, and Teen-LABS studies (Table 2). Refinement of micronutrition supply during the follow-up after bariatric surgery was commonly recommended for both study groups. The implementation of well-designed regimens during closer follow-up is essential, and the specific challenges associated with continuous adherence in adolescents are important. ${ }^{13,16}$ To date, although the validity of bariatric surgical proce- 
Table 2. Micronutrient deficiencies $>2$ to 5 years after RYGB

\begin{tabular}{llcccrrr}
\hline Author (year) & \multicolumn{1}{c}{ Study } & Procedure, patient (n) & Follow-up period (yr) & Anemia & Low ferritin or iron & Low vitamin B12 & Vitamin D deficiency \\
\hline Olbers et al. (2017)16 & AMOS & RYGB, 81 & $>5$ & $25 / 77(32)$ & $51 / 77(66)$ & $16 / 73(22)$ & $20 / 73(27)$ \\
Inge et al. (2017) & FABS 5+ & RYGB, 58 & $>5$ & $25 / 58(43)$ & $35 / 51(69)$ & $8 / 50(16)$ & $22 / 48(46)$ \\
Inge et al. (2019) & RYGB, 161 & $>2$ & NA & $83 / 127(65)$ & $10 / 121(8)$ & $61 / 128(48)$ \\
& Teen-LABS & RG, 67 & & & $15 / 44(34)$ & $3 / 39(8)$ & $13 / 44(30)$ \\
\hline
\end{tabular}

Values are presented as number/total (\%).

RYGB, Roux-en-Y gastric bypass; AMOS, Swedish Adolescent Morbid Obesity Surgery; FABS 5+, Follow-up of Adolescent Bariatric Surgery at 5 Plus Years; Teen-LABS, Teen-Longitudinal Assessment of Bariatric Surgery; SG, sleeve gastrectomy; NA, not available.

dures for adolescents has been confirmed, the probability of postoperative micronutrient deficiency adversely affecting growth, maturation, or development in adolescents warrants further exploration.

Currently, surgeons tend to prefer SG, which is gaining popularity over the last 5 years due to fewer complications and similar outcomes to RYGB. ${ }^{14,29,30}$ In a recent study, no intraoperative complications were reported, however, one case of postoperative pancreatitis occurred among adolescents with median BMI $>50 \mathrm{~kg} / \mathrm{m}^{2}$ who underwent SG. ${ }^{31}$ Additional data from 108 adolescents between the ages of 5 and 21 years who received bariatric surgery showed a $4.3 \%$ rate of minor (without major) complications..$^{32}$ More recently, accredited bariatric centers reported a 93\% uneventful recovery rate in adolescents but a $<2 \%$ immediate postoperative complication rate. An uneventful event was $>2$ times likely to occur after SG compared with RYGB after adjustment for risk factors. ${ }^{25}$ The notable shift to SG in adolescent bariatric surgery is attributable to those findings. ${ }^{33}$

\section{TIMING OF BARIATRIC SURGERY}

The optimal timing of bariatric surgery for adolescents with severe obesity remains unclear. Almost a decade ago, Inge et al..$^{28}$ raised the concern of optimal timing to achieve effective weight loss and comorbidity resolution and prevention. The authors addressed this question in the early part of their stepwise studies, in which a rational argument was suggested based on the outcomes in the Teen-LABS study. ${ }^{28}$ The authors stated that preoperative BMI was a major determinant of nadir BMI after RYGB in adolescents. Michalsky et al. ${ }^{20}$ reported the relevant data indicated that sustained weight reduction was more likely in patients with relatively lower $\left(<50 \mathrm{~kg} / \mathrm{m}^{2}\right)$ baseline BMIs who were generally younger. Further- more, Inge et al. ${ }^{13}$ ascertained that most study participants with an excessively high BMI remained obese even though they lost a significant amount of weight postoperatively, which appears attributable to the floor effect of bariatric surgery. This finding supports the hypothesis that surgical intervention in the earlier phase of obesity is associated with a better chance of obtaining normal weight. The idea that earlier intervention leads to more significant beneficial effects may also influence resolution of obesity-associated comorbidities, a concept previously proposed by Michalsky et al. ${ }^{20}$ The authors investigated the changes in risk factors of cardiovascular disorders, such as hypertension and dyslipidemia, during a 3-year follow-up after RYGB and SG. Overall, the patients with at least one cardiovascular risk factor decreased from $97 \%$ at baseline to only $48 \% 3$ years after surgery. Preoperatively, more than three different cardiovascular risk factors occurred in 33\% of participants and in only $5 \% 3$ years after bariatric surgery. Patients who did not have definite dyslipidemia or hypertension at baseline also showed significant improvements in lipid profile and blood pressure values over time..$^{20}$ Because the ability to normalize high blood pressure and dyslipidemia in adolescent patients who received bariatric surgery decreased in patients with higher BMIs, bariatric surgery at lower thresholds for intervention will hypothetically be much more beneficial.

Earlier surgical intervention can also be justified because the increase in risk of various metabolic derangements can be attenuated in patients who achieve successful weight reduction through nonsurgical options before reaching adolescence. ${ }^{34}$ Children with obesity who remained obese in adulthood had increased risks of obesity-related disorders, including T2DM, high blood pressure, and dyslipidemia. The risk of exhibiting these comorbidities in adulthood was similar to people who were never obese. ${ }^{34}$ However, Bjerregaard et al. ${ }^{35}$ reported that patients who were overweight in child- 
hood ( $<13$ years) but not in adulthood, had a higher risk of comorbidities (hazard ratio, 1.47; $P<0.05$ ) in adulthood, but this risk was not as high as in subjects who were still obese in adulthood. The data may support the potential benefits of resolving obesity by bariatric surgery earlier in adolescence to prevent obesity-related comorbidities in adulthood and further reinforce the practice of considering bariatric surgery for adolescents with BMIs $>40$ or $>35 \mathrm{~kg} / \mathrm{m}^{2}$ with specific comorbidities. The concept that early bariatric surgery in adolescents without an extremely high BMI could provide better outcomes is well-justified.

The main findings from the latest Teen-LABS ( > 5 years' followup) study showed a more favorable improvement in hypertension and T2DM among adolescents compared with adults. ${ }^{18}$ Based on the results from the Teen-LABS study, adolescents have a greater chance of achieving remission of T2DM ( $86 \%$ vs. 53\%; risk ratio, 1.27 ) and resolution of hypertension ( $68 \%$ vs. $41 \%$; risk ratio, 1.51 ) compared with adults after bariatric surgery, whereas the magnitude of weight reduction ( $26 \%$ vs. $29 \%$; $P>0.05$ ) did not differ between adolescents and adults. Bariatric surgery early after diagnosis was hypothesized to have influenced the favorable outcomes of T2DM remission based on the so-called salvage phenomenon in pancreatic beta-cell insulin secretory function before the irreversible injury. In general, T2DM is more aggressive in adolescents than in adults which leads to a higher failure rate of first-line oral anti-hyperglycemic agents. ${ }^{36,37}$ Nevertheless, bariatric surgery has successfully shown encouraging evidence that RYGB, and currently SG, could be reliable therapeutic options for adolescent T2DM patients if weight reduction is sustained, based on the long-term data from the Teen-LABS, FABS 5+, and AMOS studies.

\section{CONCLUSION}

The sustainability of weight loss and comorbidity resolution using bariatric surgery in severely obese adolescents is well supported by recent multicenter prospective long-term follow-up data. A specialized pediatric care team should be involved in the evaluation process while considering the relevant age-related issues, including immediate and long-term postoperative complications associated with the modification of surgical anatomy, physical development, and compliance. Regarding the timing of intervention, earlier sur- gery in adolescents without an extremely high BMI can increase the likelihood of a healthier life. Therefore, strict guidelines should be established for adolescent bariatric surgery based on scientific understanding of the sustainable effects and in consideration of any possible age-related adverse effects.

\section{CONFLICTS OF INTEREST}

The author declares no conflict of interest.

\section{REFERENCES}

1. Hales CM, Fryar CD, Carroll MD, Freedman DS, Ogden CL. Trends in obesity and severe obesity prevalence in US youth and adults by sex and age, 2007-2008 to 2015-2016. JAMA 2018;319:1723-25.

2. Skinner AC, Perrin EM, Skelton JA. Prevalence of obesity and severe obesity in US children, 1999-2014. Obesity (Silver Spring) 2016;24:1116-23.

3. Reilly JJ, Kelly J. Long-term impact of overweight and obesity in childhood and adolescence on morbidity and premature mortality in adulthood: systematic review. Int J Obes (Lond) 2011;35:891-8.

4. Zambrano E, Ibáñez C, Martínez-Samayoa PM, Lomas-Soria C, Durand-Carbajal M, Rodríguez-González GL. Maternal obesity: lifelong metabolic outcomes for offspring from poor developmental trajectories during the perinatal period. Arch Med Res 2016;47:1-12.

5. Sarno LA, Lipshultz SE, Harmon C, De La Cruz-Munoz NF, Balakrishnan PL. Short- and long-term safety and efficacy of bariatric surgery for severely obese adolescents: a narrative review. Pediatr Res 2020;87:202-9.

6. Must A, Jacques PF, Dallal GE, Bajema CJ, Dietz WH. Longterm morbidity and mortality of overweight adolescents: a follow-up of the Harvard Growth Study of 1922 to 1935 . N Engl J Med 1992;327:1350-5.

7. Rajendram R, Preedy VR. Laparoscopic adjustable gastric banding. In: Preedy V, Rajendram R, Martin C, editors. Metabolism and pathophysiology of bariatric surgery: nutrition, procedures, outcomes and adverse effects. London: Academic Press; 2016. 
p. 113-7.

8. Inge TH, Laffel LM, Jenkins TM, Marcus MD, Leibel NI, Brandt ML, et al. Comparison of surgical and medical therapy for type 2 diabetes in severely obese adolescents. JAMA Pediatr 2018; $172: 452-60$

9. Park KH. Intervention for severely obese children and adolescents. J Obes Metab Syndr 2019;28:1-3.

10. Vanguri P, Lanning D, Wickham EP, Anbazhagan A, Bean MK. Pediatric health care provider perceptions of weight loss surgery in adolescents. Clin Pediatr (Phila) 2014;53:60-5.

11. Messiah SE, Lipshultz SE, Natale RA, Miller TL. The imperative to prevent and treat childhood obesity: why the world cannot afford to wait. Clin Obes 2013;3:163-71.

12. Lawson ML, Kirk S, Mitchell T, Chen MK, Loux TJ, Daniels SR, et al. One-year outcomes of Roux-en-Y gastric bypass for morbidly obese adolescents: a multicenter study from the Pediatric Bariatric Study Group. J Pediatr Surg 2006;41:137-43.

13. Inge TH, Courcoulas AP, Jenkins TM, Michalsky MP, Helmrath MA, Brandt ML, et al. Weight loss and health status 3 years after bariatric surgery in adolescents. N Engl J Med 2016;374: 113-23.

14. Barnett SJ. Surgical management of adolescent obesity. Adv Pediatr 2013;60:311-25.

15. Reinehr T. Long-term effects of adolescent obesity: time to act. Nat Rev Endocrinol 2018;14:183-8.

16. Olbers T, Beamish AJ, Gronowitz E, Flodmark CE, Dahlgren J, Bruze G, et al. Laparoscopic Roux-en-Y gastric bypass in adolescents with severe obesity (AMOS): a prospective, 5-year, Swedish nationwide study. Lancet Diabetes Endocrinol 2017; 5:174-83.

17. Inge TH, Jenkins TM, Xanthakos SA, Dixon JB, Daniels SR, Zeller $\mathrm{MH}$, et al. Long-term outcomes of bariatric surgery in adolescents with severe obesity (FABS-5+): a prospective follow-up analysis. Lancet Diabetes Endocrinol 2017;5:165-73.

18. Inge TH, Courcoulas AP, Jenkins TM, Michalsky MP, Brandt ML, Xanthakos SA, et al. Five-year outcomes of gastric bypass in adolescents as compared with adults. N Engl J Med 2019; 380:2136-45.

19. Mingrone G, Panunzi S, De Gaetano A, Guidone C, Iaconelli A, Nanni G, et al. Bariatric-metabolic surgery versus conventional medical treatment in obese patients with type 2 diabetes: 5 year follow-up of an open-label, single-centre, randomised controlled trial. Lancet 2015;386:964-73.

20. Michalsky MP, Inge TH, Jenkins TM, Xie C, Courcoulas A, Helmrath $\mathrm{M}$, et al. Cardiovascular risk factors after adolescent bariatric surgery. Pediatrics 2018;141:e20172485.

21. Wasserman $H$, Inge TH. Bariatric surgery in obese adolescents: opportunities and challenges. Pediatr Ann 2014;43:e230-6.

22. Inge TH, Coley RY, Bazzano LA, Xanthakos SA, McTigue K, Arterburn D, et al. Comparative effectiveness of bariatric procedures among adolescents: the PCORnet bariatric study. Surg Obes Relat Dis 2018;14:1374-86.

23. DuCoin C, Moon RC, Mulatre M, Teixeira AF, Jawad MA. Safety and effectiveness of Roux-en-Y gastric bypass in patients between the ages of 17 and 19. Obes Surg 2015;25:464-9.

24. Serrano OK, Zhang Y, Kintzer E, Moran-Atkin E, Choi J, Melvin WS, et al. Outcomes of bariatric surgery in the young: a single-institution experience caring for patients under 21 years old. Surg Endosc 2016;30:5015-22.

25. Inge TH, Zeller MH, Jenkins TM, Helmrath M, Brandt ML, Michalsky MP, et al. Perioperative outcomes of adolescents undergoing bariatric surgery: the teen-longitudinal assessment of bariatric surgery (Teen-LABS) study. JAMA Pediatr 2014; 168:47-53.

26. Altieri MS, Pryor A, Bates A, Docimo S, Talamini M, Spaniolas K. Bariatric procedures in adolescents are safe in accredited centers. Surg Obes Relat Dis 2018;14:1368-72.

27. Arafat M, Norain A, Burjonrappa S. Characterizing bariatric surgery utilization and complication rates in the adolescent population. J Pediatr Surg 2019;54:288-92.

28. Inge TH, Jenkins TM, Zeller M, Dolan L, Daniels SR, Garcia $\mathrm{VF}$, et al. Baseline BMI is a strong predictor of nadir BMI after adolescent gastric bypass. J Pediatr 2010;156:103-8.

29. Kumar S, Kelly AS. Review of childhood obesity: from epidemiology, etiology, and comorbidities to clinical assessment and treatment. Mayo Clin Proc 2017;92:251-65.

30. Goldberg HR, Chin VL, Zitsman JL, Zhang C, Williams KM, Oberfield S, et al. Bariatric surgery in adolescents: is routine nutrient supplementation sufficient to avoid anemia following bariatric surgery? Nutr Clin Pract 2017;32:502-7. 
31. McGuire MM, Nadler EP, Qureshi FG. Laparoscopic vertical sleeve gastrectomy for adolescents with morbid obesity. Semin Pediatr Surg 2014;23:21-3.

32. Alqahtani AR, Antonisamy B, Alamri H, Elahmedi M, Zimmerman VA. Laparoscopic sleeve gastrectomy in 108 obese children and adolescents aged 5 to 21 years. Ann Surg 2012;256: 266-73.

33. Chernoguz A, Chwals WJ. Bariatric surgery needs a seat at the children's table: bridging the perception and reality of the role of bariatric surgery in the treatment of obesity in adolescents. Clin Ther 2018;40:1648-54.

34. Juonala M, Magnussen CG, Berenson GS, Venn A, Burns TL,
Sabin MA, et al. Childhood adiposity, adult adiposity, and cardiovascular risk factors. N Engl J Med 2011;365:1876-85.

35. Bjerregaard LG, Jensen BW, Ängquist L, Osler M, Sørensen TIA, Baker JL. Change in overweight from childhood to early adulthood and risk of type 2 diabetes. N Engl J Med 2018; 378:1302-12.

36. Kahn SE, Haffner SM, Heise MA, Herman WH, Holman RR, Jones NP, et al. Glycemic durability of rosiglitazone, metformin, or glyburide monotherapy. N Engl J Med 2006;355:2427-43.

37. Beamish AJ, D’Alessio DA, Inge TH. Controversial issues: when the drugs don't work, can surgery provide a different outcome for diabetic adolescents? Surg Obes Relat Dis 2015;11:946-8. 